\title{
Development of Ni particle dispersed poly(methylmethacrylate) composites exhibiting conductor/insulator transition by the positive temperature coefficient effect of electrical resistivity
}

\author{
Polymer Journal (2013) 45, 690-694; doi:10.1038/pj.2012.223; published online 23 January 2013
}

\section{INTRODUCTION}

A number of conductive polymer composites composed of crystalline polymer filled with carbon black, ${ }^{1-5}$ carbon nanotubes ${ }^{6}$ and metal particles ${ }^{7-11}$ as conductive fillers exhibit the positive temperature coefficient (PTC) effect of electrical resistivity. The PTC effect implies that resistivity increases with rising temperature. The PTC intensity (peak resistivity $\rho_{\mathrm{H}}$ /resisitivity at room temperature $\rho_{\mathrm{L}}$ ) of some composites is greater than $10^{7}$, and composites demonstrate conductor/ insulator transition with changing temperature. Therefore, some composites are usable for self-recovering fuses for electronic circuits.

The PTC effect of composites composed of crystalline polymer is explained by the following model. The resistivity of almost pure polymer without conductive filler exceeds $10^{12} \Omega \mathrm{cm}$, and these polymers behave similar to insulators. When conductive filler content reaches a specific value, the resistivity of composites demonstrates a sudden decrease from insulating range to conducting range because the conductive filler is joined together similar to a network, forming a conductive path. Some composites with many metal particles exhibit a low $\rho_{\mathrm{L}}$ of $10^{-2} \Omega \mathrm{cm} .{ }^{8-10}$ These characteristics are known as the percolation phenomenon. ${ }^{12-16}$ However, the resistivity of composites exceeds $10^{9} \Omega \mathrm{cm}^{8-10}$ at near the melting point of the matrix polymer even with a large amount of conductive filler. This resistivity change is caused by a disconnection of conductive paths following the drastic volume expansion of matrix polymer due to melting. ${ }^{2}$ Therefore, a number of composites composed of crystalline polymer exhibit the PTC effect.

The PTC effect of composites was explained by the above models, and so it was assumed that composites composed of amorphous polymer, which do not exhibit the melting phenomenon, do not exhibit the PTC effect. In fact, it was reported that poly(methylmethacrylate) (PMMA)/carbon black composites do not demonstrate the PTC effect, because, unlike crystalline polymers, amorphous polymers do not exhibit drastic volume expansion. $^{2}$ Moreover, it was reported that the PTC intensity of polystyrene/carbon black composites is only in double digits. ${ }^{5}$

In this study, we investigated the influence of Ni content on the PTC effect of Ni particle dispersed PMMA composites. Composites with Ni contents of 20 and 25 vol.\% exhibited the PTC effect. Moreover, the PTC intensity of a composite with a $\mathrm{Ni}$ content of 25 vol.\% reached $10^{8}$, a value equivalent to that of composites composed of crystalline polymer.

\section{EXPERIMENTAL PROCEDURE}

PMMA (HBS000, Mitsubishi Rayon, Tokyo, Japan), linear low-density polyethylene (LLDPE: Evolue 3010, Prime Polymer, Tokyo, Japan) and poly(vinylidene fluoride) (PVDF: KYNAR K720, Arkema, Colombes, France) were used as a matrix polymer of the composites. Ni particles manufactured by Inco (Toronto, Canada) were used as the conductive filler. Its average particle size was $2.5 \mu \mathrm{m}$, and its resistivity was $7 \times 10^{-5}$ $\Omega \mathrm{cm}$. Polymer pellets and $\mathrm{Ni}$ particles were meltblended at $200{ }^{\circ} \mathrm{C}$ and 60 r.p.m. for $15 \mathrm{~min}$, using a two-roller mixer (4M150, Toyo Seiki, Tokyo, Japan). The composite was inserted between two steel boards $(150 \mathrm{~mm} \times 150 \mathrm{~mm} \times 2.2 \mathrm{~mm})$ and molded to a 1-mm thick sheet (sample) using the hot-press method (G-12, TECHNO SUPPLY, Tokyo, Japan). Hot-press was performed at $10 \mathrm{MPa}$ and $200^{\circ} \mathrm{C}$ for $7 \mathrm{~min}$. The sample between two steel boards was then cooled in air.

The PTC effect was evaluated by measuring resistance using the two-terminal method, whereas the sample was heated in an oven (DRX 320DA, Advantec, Tokyo, Japan) at a rate of $1{ }^{\circ} \mathrm{C} \mathrm{min}{ }^{-1}$. A digital multimeter (PC520M, Sanwa, Tokyo, Japan) was used to measure resistance. In the two-terminal method, resistivity $\rho$ was calculated using the equation $\rho=R S / d$ from resistance $R$, sample area $S$ and sample thickness $d$. The sample area was $1 \mathrm{~cm}^{2}$, and the sample thickness was $0.8 \mathrm{~mm}$. Ni foil was heat-sealed at $5 \mathrm{MPa}$ and $200{ }^{\circ} \mathrm{C}$ for $5 \mathrm{~min}$ to form electrodes on both sides of the sample. After Ni foil lamination, the sample was cooled in air.

The aggregate structure of $\mathrm{Ni}$ particles was observed by scanning electron microscope (SEM: TM-1000, Hitachi High-Technologies, Tokyo, Japan). Composites were fractured at room temperature, and the sectional area was observed. The GNOMIX PVT apparatus (Gnomix, CO, USA) was used to measure the specific volume of pure PMMA from room temperature to $200^{\circ} \mathrm{C}$, and at pressures between 10 and $200 \mathrm{MPa}$ in steps of $10 \mathrm{MPa}$. Data were extrapolated to zero pressure.

\section{RESULTS AND DISCUSSION}

Figure 1 plots the PTC curves of PMMA/Ni composites with Ni contents of 20, 25, 30, 35 and 40 vol. $\%$. The $\rho_{\mathrm{L}}$ decreased with increasing $\mathrm{Ni}$ content, which is consistent with percolation. $^{8,10,12-16}$ The resistivity of composites with $\mathrm{Ni}$ contents of 20 and 25 vol.\% increased drastically with rising temperature, and these composites exhibited the PTC effect. These composites exhibited constant values of $10^{9} \Omega \mathrm{cm}$ of $\rho_{\mathrm{H}}$ because 
they reached the upper measurement limit of the digital multimeter. Therefore, it is assumed that the PTC intensity of a composite with the Ni content of 20 vol.\% exceeds $10^{4}$, and that of a composite with the $\mathrm{Ni}$ content of 25 vol.\% exceeds $10^{8}$. These high PTC intensities of composites composed of amorphous polymer have not yet been reported and are a unique finding among the numerous studies. However, in composites with $\mathrm{Ni}$ contents of 30,35 and 40 vol.\%, resistivity was maximum at $90^{\circ} \mathrm{C}$. In these composites, $\rho_{\mathrm{H}}$ and slopes of PTC curves decreased with increasing $\mathrm{Ni}$ content. Moreover, $\rho_{\mathrm{H}}$ and slopes of PTC curves in these composites were much smaller than those of composites with $\mathrm{Ni}$ contents of 20 and 25 vol.\%. Therefore, we conclude that composites with $\mathrm{Ni}$ contents of 30,35 and 40 vol.\% do not exhibit the PTC effect.

The mechanism of the PTC effect for PMMA/Ni composites that do not exhibit

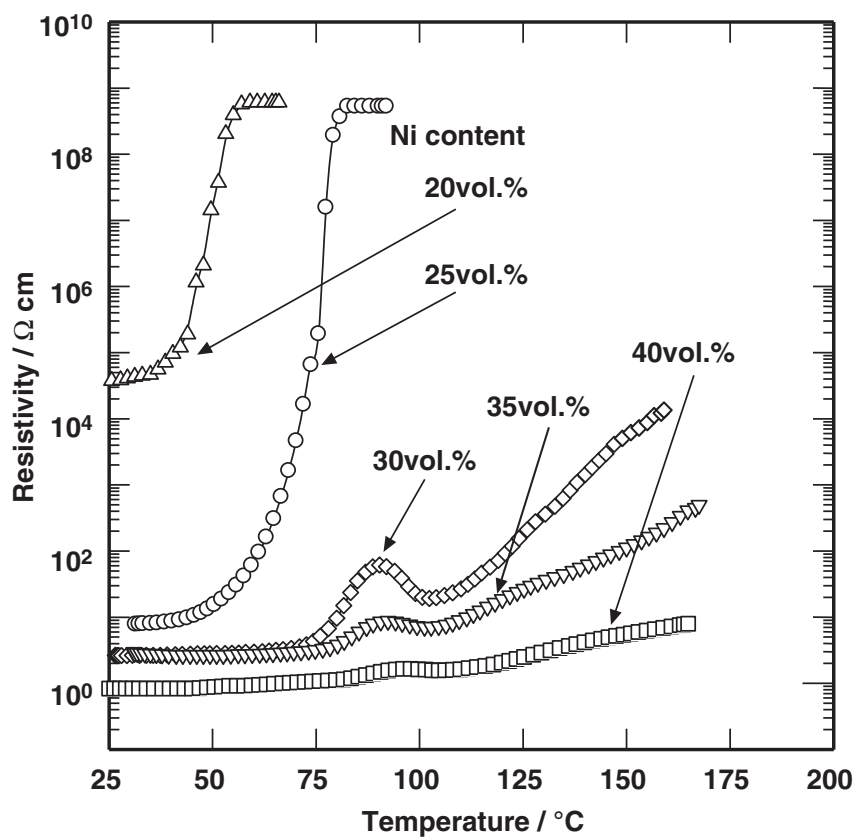

Figure 1 PTC curves of PMMA/Ni composites with various Ni contents.

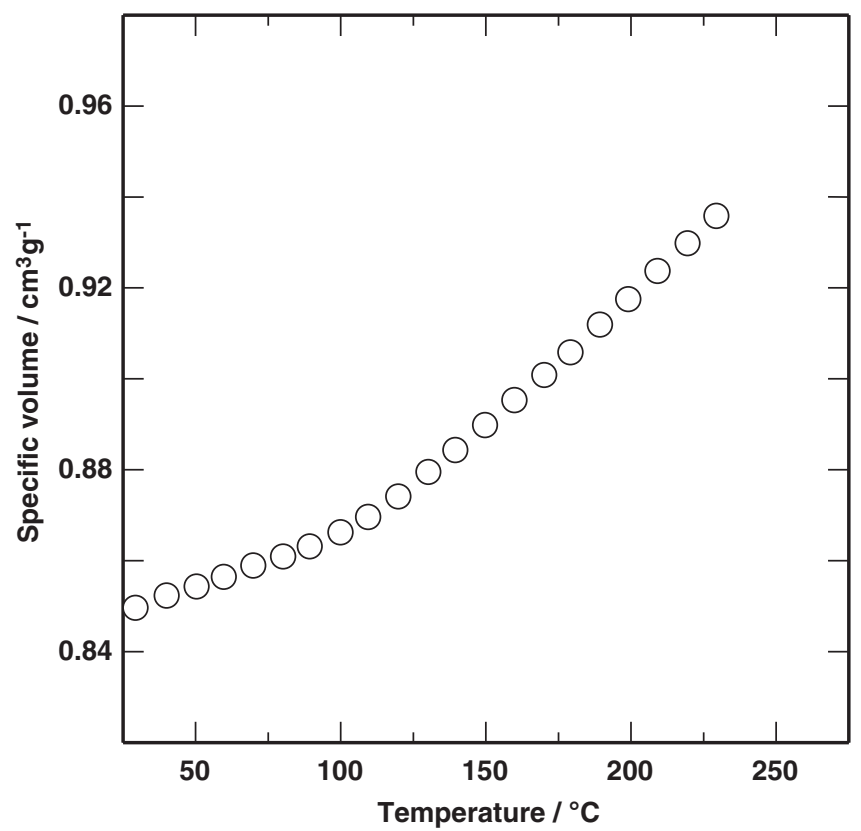

Figure 2 Pressure-volume-temperature curve of PMMA at the pressure of O MPa.

melting is discussed in detail below. One theory regarding the PTC effect in composites is the thermal-fluctuation-induced tunneling theory by Sheng et al. ${ }^{2,8,17}$ Currently, it is assumed that the electrical conduction mechanism of composites is tunneling conduction between fillers. According to this theory, resistivity depends exponentially on the gap length between fillers. ${ }^{2,8}$ That is, electron transfer from a filler to the nearest neighboring filler decreases because the tunneling gap width (potential barrier width) increases with increasing gap length between fillers. Therefore, resistivity increases drastically with a slight increase of gap length between fillers. To clarify the factor affecting the gap length between fillers in PMMA/Ni composites, we evaluated the pressurevolume-temperature behavior of PMMA. Figure 2 plots the pressure-volume-temperature curve of PMMA at $0 \mathrm{MPa}$. No significant change in specific volume due to melting (for example, crystalline polymer) was observed in PMMA, but the specific volume of PMMA increased slightly with rising temperature. If a gap between $\mathrm{Ni}$ particles is generated by increased specific volume in PMMA, it is reasonable to assume that $\mathrm{PMMA} / \mathrm{Ni}$ composites exhibit the PTC effect, following the fluctuation-induced tunneling model.

We seek to determine why only PMMA/Ni composites with $\mathrm{Ni}$ contents of 20 and 25 vol.\% exhibit the PTC effect. First, we analyze the cluster structures forming fillers (that is, conductive paths) for PMMA/Ni composites with various $\mathrm{Ni}$ contents. Figure 3 presents SEM images of PMMA/Ni composites with $\mathrm{Ni}$ contents of 25 and 40 vol.\%. Ni particles were dispersed uniformly in the PMMA matrix, and the cluster composed of $\mathrm{Ni}$ particles in composites with a Ni content of 25 vol.\% was smaller than that of those with 40 vol.\%. In our previous studies on the influence of $\mathrm{Ni}$ content on electrical properties of LLDPE/Ni composites, ${ }^{10}$ high-density polyethylene/Ni composites $^{9}$ and PVDF/Ni composites, ${ }^{8}$ we found that the number of conductive paths decreases with decreasing $\mathrm{Ni}$ content. Moreover, Wu et $a l^{2}$ analyzed the lattice model and demonstrated that the gap length between carbon black particles in high-density polyethylene/carbon black composites increases with decreasing carbon black content.

Second, we determine the disconnection of conductive paths (that is, onset of the PTC effect) for PMMA/Ni composites with various $\mathrm{Ni}$ contents. Ni content dependence on the PTC effect in the PMMA/Ni composites can be explained in terms of the following 

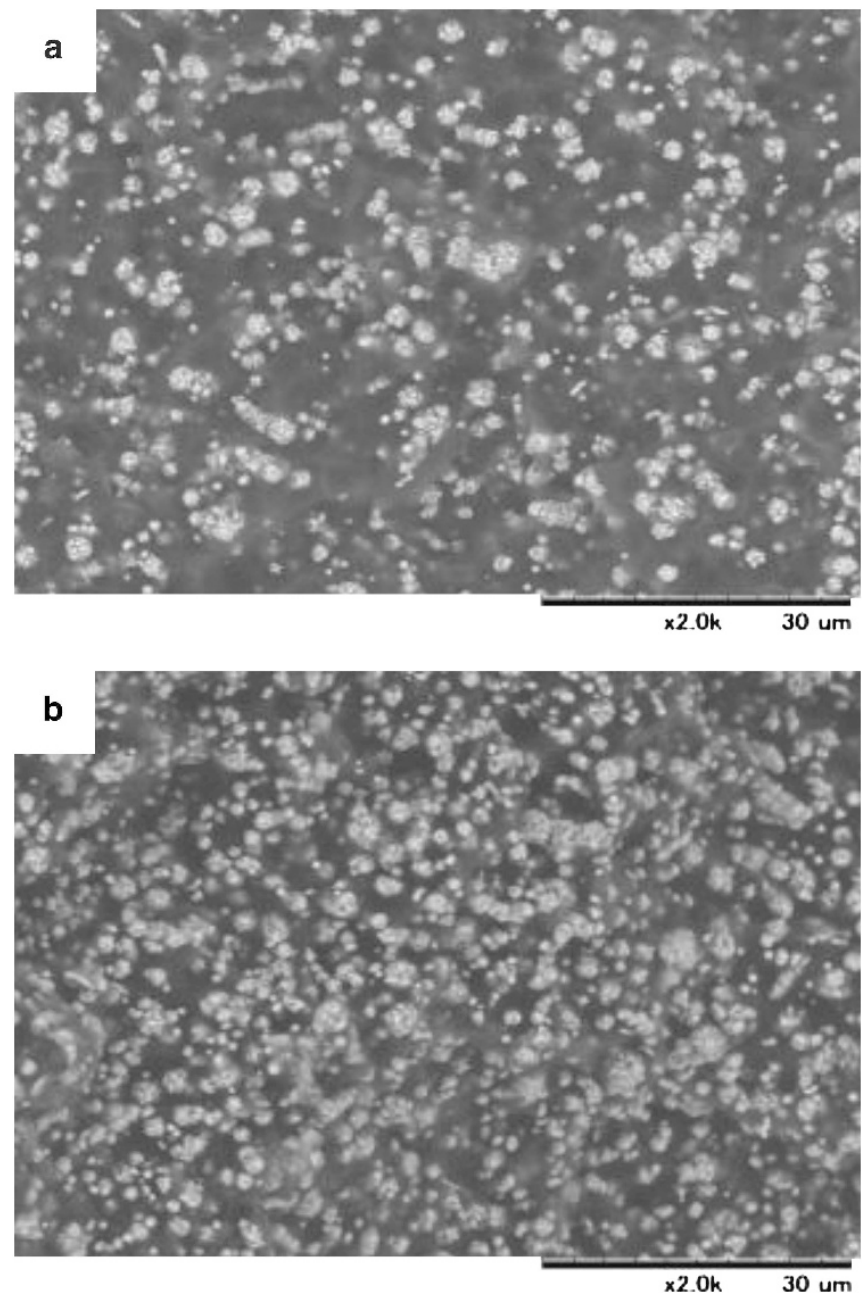

Figure 3 SEM images $(\times 2000)$ of PMMA/Ni composites. (a) Ni content $=25$ vol.\% (highest PTC intensity). (b) Ni content $=40$ vol.\% (no PTC effect).

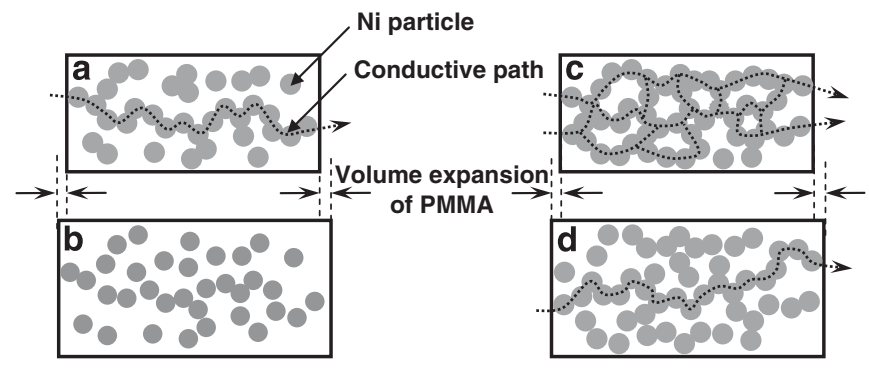

Figure 4 Schematic illustrations of the change in Ni particle connections. (a) Composite with a few $\mathrm{Ni}$ particles at low temperature. (b) Composite with a few Ni particles at high temperature. (c) Composite with many Ni particles at low temperature. (d) Composite with many Ni particles at high temperature.

model. Figure $4 \mathrm{a}-\mathrm{d}$ schematically illustrate the conductive path of the PMMA/Ni composite estimated from SEM observation and previous studies. ${ }^{8-10}$ Fewer conductive paths exist when $\mathrm{Ni}$ content is low (Figure $4 \mathrm{a}$ ). Therefore, it is assumed that a small gap between $\mathrm{Ni}$ particles is generated even with slight volume expansion of PMMA, due to temperature rising (Figure $4 \mathrm{~b}$ ). According to the fluctuation-induced tunneling model, the resistivity of composites increases drastically with the generation of even a small gap between fillers. Thus, PMMA/Ni composites with $\mathrm{Ni}$ contents of 20 and 25 vol.\% exhibit the PTC effect. However, the number of conductive paths increases with increasing $\mathrm{Ni}$ content. Composites with high Ni content have large network-like conductive paths (Figure 4c). Therefore, a gap between $\mathrm{Ni}$ particles is difficult to generate with a slight volume expansion of PMMA. In addition, if the gap between $\mathrm{Ni}$ particles is generated in part of the network but contact is maintained in the other parts, the conductive path is maintained (Figure 4d). Thus, composites with $\mathrm{Ni}$ contents of 30,35 and 40 vol.\% exhibit low PTC curve slopes and no PTC effect.

Resistivity is maximum at $90{ }^{\circ} \mathrm{C}$ with $\mathrm{Ni}$ contents of 30, 35 and 40 vol.\% (Figure 1). The molecular motion of PMMA becomes active near the glass transition point of $105^{\circ} \mathrm{C}$. We assume that the positions of the $\mathrm{Ni}$ particles change momentarily with the molecular motion of PMMA, and the conductive path disconnects temporarily. However, it is assumed that the $\mathrm{Ni}$ particles reassemble when PMMA becomes rubbery and the conductive path increases. ${ }^{18}$ The result is a maximum in PTC curves. This phenomenon should occur even with composites with $\mathrm{Ni}$ contents of 20 and 25 vol.\%. However, this phenomenon could not be observed because the PTC effect increases the resistivity of these composites.

Figure $5 \mathrm{a}$ and $\mathrm{b}$ present the PTC curves of $\mathrm{PVDF} / \mathrm{Ni}$ and LLDPE/Ni composites. All $\mathrm{PVDF} / \mathrm{Ni}$ and LLDPE/Ni composites exhibited the PTC effect. The onset temperature of the PTC effect in these composites rose with increasing $\mathrm{Ni}$ content. It is assumed that the number of conductive paths increases with increasing $\mathrm{Ni}$ content. Therefore, with increasing $\mathrm{Ni}$ content, it becomes more difficult to disconnect a conductive path by increasing the specific volume of the composite. To disconnect a conductive path, a greater increase in the specific volume of the composite is necessary. Thus, we conclude that the onset of the PTC effect occurs later with increasing $\mathrm{Ni}$ content because the specific volume of the composite increases at higher temperatures. For details, refer to our previous works.. ${ }^{8-10}$

Table 1 indicates $\rho_{\mathrm{L}}, \rho_{\mathrm{H}}$ and the order of PTC intensity for various composites. When the $\mathrm{Ni}$ content was $20 \mathrm{vol} . \%$, the $\rho_{\mathrm{H}}$ of PMMA/Ni composites was comparable to that of PVDF/Ni composites, whereas the $\rho_{\mathrm{L}}$ of PMMA/Ni composites was higher than that of PVDF/Ni composites. Therefore, the PTC intensity of PMMA/Ni composites was less than that of PVDF/Ni composites. The crystalline polymer crystallizes, excluding extraneous materials such as filler. As a result, 

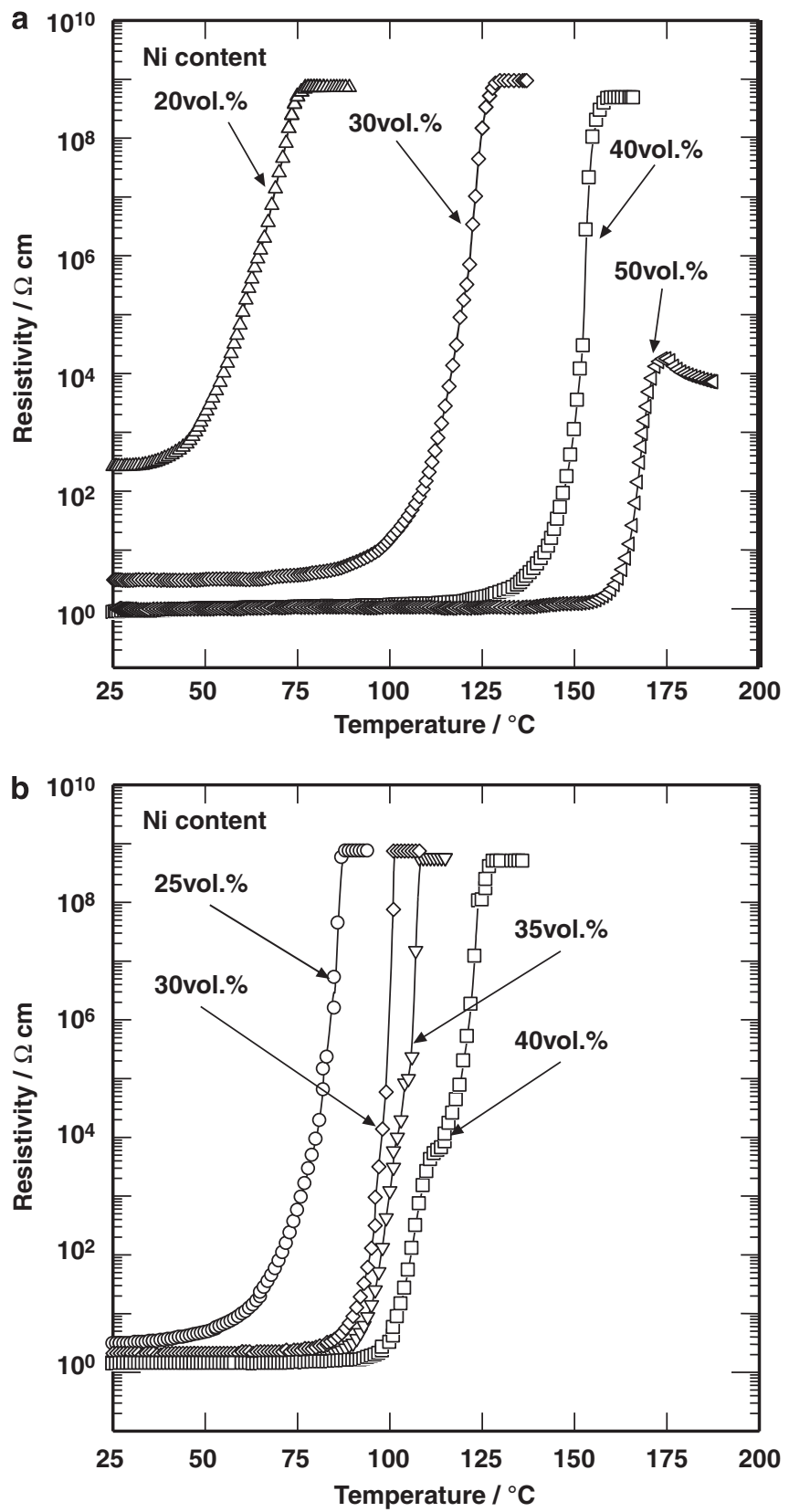

Figure 5 PTC curves of (a) PVDF/Ni composites and (b) LLDPE/Ni composites with various $\mathrm{Ni}$ contents.

it is assumed that the fillers are concentrated spatially with crystallization of the matrix polymer; thus, the conductive path connects easily. ${ }^{9,10}$ However, in the composites composed of amorphous polymer, fillers are dispersed uniformly in the matrix polymer; thus, development of conductive paths becomes difficult. These factors lead to high $\rho_{\mathrm{L}}$ following low PTC intensity in the $\mathrm{PMMA} / \mathrm{Ni}$ composites. However, when the Ni content was from 30 to $40 \mathrm{vol} . \%$, the $\rho_{\mathrm{L}}$ of $\mathrm{PMMA} / \mathrm{Ni}$ composites was comparable to that of $\mathrm{PVDF} / \mathrm{Ni}$ and LLDPE/Ni composites, whereas the $\rho_{\mathrm{H}}$ of PMMA/Ni composites was smaller than that of PVDF/ $\mathrm{Ni}$ and LLDPE/Ni composites. Therefore, PTC intensity for PMMA/Ni composites was less than that for $\mathrm{PVDF} / \mathrm{Ni}$ and LLDPE/Ni composites because the volume expansion of PMMA, which does not exhibit melting, is less than that of PVDF and LLDPE. Table 1 indicates that PMMA/Ni composites exhibit a PTC intensity of $10^{8}$ or more when the Ni content is 25 vol.\%.
This PTC intensity is comparable to those of $\mathrm{PVDF} / \mathrm{Ni}$ and LLDPE/Ni composites. It is assumed that the Ni content of 25 vol.\% in PMMA/Ni composites strikes a balance between development of conductive paths and disconnection of conductive paths by a slight volume expansion. These results indicate that the most important factor for the onset of the PTC effect in PMMA/Ni composites is Ni content.

Until now, carbon black was generally used as a filler for composites. ${ }^{1-5}$ It is assumed that carbon black particles adhere strongly to each other because the carbon black surface has microasperity. Therefore, it would be difficult to disconnect conductive paths in polymer/carbon black composites, and so it may well be that composites composed of amorphous polymers with only a slight volume expansion did not exhibit the PTC effect in previous studies. ${ }^{2}$ Moreover, both the coefficient of volume expansion and the glass transition point of amorphous polymers should be noted. If the coefficient of volume expansion is small, composites should not exhibit the PTC effect. In addition, the shape of the PTC curve is affected by the glass transition point, that is, the temperature at the maximum of the PTC curve might change. These results indicate that if the kinds of polymer, filler and filler content are optimized, composites composed of amorphous polymer, which do not have the melting phenomenon, can be expected to exhibit a PTC effect comparable to that of composites composed of crystalline polymer.

\section{CONCLUSIONS}

We investigated the influence of $\mathrm{Ni}$ content on the PTC effect of Ni particle dispersed PMMA composites. PMMA/Ni composites with $\mathrm{Ni}$ contents of 20 and 25 vol.\% exhibit the PTC effect, whereas PMMA/Ni composites with Ni contents of 30, 35 and 40 vol.\% do not exhibit the PTC effect. The PTC intensity of a composite with a $\mathrm{Ni}$ content of 25 vol. $\%$ was equivalent to that of composites composed of crystalline polymer (for example, PVDF and LLDPE). In composites with Ni contents of 20 and 25 vol.\%, there was little contact between Ni particles, so a gap between $\mathrm{Ni}$ particles was generated even with slight volume expansion of PMMA and the PTC effect occurred. However, in composites with $\mathrm{Ni}$ contents of 30, 35 and 40 vol.\%, the contact between $\mathrm{Ni}$ particles was strong, and so no gap was generated between $\mathrm{Ni}$ particles with a slight volume expansion of PMMA. Previously, it was assumed that composites composed of amorphous polymer, which do not exhibit 
Table $1 \rho_{\mathrm{L}}, \rho_{\mathrm{H}}$ and PTC intensity of various composites

\begin{tabular}{|c|c|c|c|c|}
\hline Composite & Ni content (vol.\%) & $\rho_{L}(\Omega c m)$ & $\rho_{H}(\Omega c m)$ & Order of PTC intensity \\
\hline \multirow[t]{5}{*}{$\mathrm{PMMA} / \mathrm{Ni}$} & 20 & 40000 & $>10^{9 a}$ & $>10^{4}$ \\
\hline & 25 & 7.8 & $>10^{9 a}$ & $>10^{8}$ \\
\hline & 30 & 2.7 & $5000^{b}$ & $10^{3}$ \\
\hline & 35 & 2.8 & $100^{b}$ & 10 \\
\hline & 40 & 1 & $5.4^{\mathrm{b}}$ & 1 \\
\hline \multirow[t]{4}{*}{$\mathrm{PVDF} / \mathrm{Ni}$} & 20 & 270 & $>10^{9 a}$ & $>10^{6}$ \\
\hline & 30 & 3.2 & $>10^{9 a}$ & $>10^{8}$ \\
\hline & 40 & 1.1 & $>10^{9 a}$ & $>10^{8}$ \\
\hline & 50 & 1.1 & 20000 & $10^{4}$ \\
\hline \multirow[t]{4}{*}{ LLDPE/Ni } & 25 & 3.2 & $>10^{9 a}$ & $>10^{8}$ \\
\hline & 30 & 2.1 & $>10^{9 a}$ & $>10^{8}$ \\
\hline & 35 & 1.6 & $>10^{9 a}$ & $>10^{8}$ \\
\hline & 40 & 1.4 & $>10^{9 a}$ & $>10^{8}$ \\
\hline
\end{tabular}

Abbreviations: PMMA, poly(methylmethacrylate); PTC, positive temperature coefficient; PVDF, poly(vinylidene fluoride). aupper measurement limit of digital multimeter.

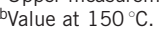

melting, do not exhibit the PTC effect with high PTC intensity. We have provided the first conclusive evidence that the PTC effect of conductive polymer composites occurs even without polymer melting.

\section{ACKNOWLEDGEMENTS}

This work was supported by JSPS KAKENHI Grant Number 24750226.

Akihiko Kono $^{1}$, Katsuya Shimizu ${ }^{2}$, Hajime Nakano ${ }^{2}$, Masashi Yamamoto ${ }^{2}$, Yousuke Goto ${ }^{2}$, Seiji Takahashi ${ }^{3}$, Toshiaki Ougizawa ${ }^{4}$ and Hideo Horibe ${ }^{2,3,5}$

${ }^{1}$ Practical Engineering Education Program, Kanazawa Institute of Technology, Nonoichi, Japan; ${ }^{2}$ Graduate School of Engineering, Kanazawa Institute of Technology, Hakusan, Japan;
${ }^{3}$ Research Laboratory for Integrated Technological Systems, Kanazawa Institute of Technology, Hakusan, Japan; ${ }^{4}$ Department of Organic and Polymeric Materials, Tokyo Institute of

Technology, Tokyo, Japan and ${ }^{5}$ The Institute of Scientific and Industrial Research, Osaka University, Ibaraki, Japan E-mail:a.kono@neptune.kanazawa-it.ac.jp

1 Horibe, H., Kamimura, T. \& Yoshida, K. Electrical conductivity of polymer composites filled with carbon black. Jpn J. Appl. Phys. 44, 2025-2029 (2005).

2 Wu, C., Suzuki, K., Asai, S., Sumita, M. \& Miyasaka, K. PTC properties of polymer composites with disperse conductive particles. TANSO 153, 149-154 (1992).

3 Wu, G., Cheng, C., Miura, T., Asai, S. \& Sumita, M Electrical characteristics of fluorinated carbon blackfilled poly(vinylidene fluoride) composites. J. Appl. Polym. Sci. 80, 1063-1070 (2001).

4 Yi, X. S., Shen, L. \& Pan, Y. Thermal volume expansion in polymeric PTC composites: a theoretical approach. Composites Sci. Technol. 61, 949-956 (2001).
5 Shen, L., Lou, Z. D. \& Qian, Y. J. Effects on thermal volume expansion on positive temperature coefficient effect for carbon black filled polymer composites. J. Polym. Sci: Part B: Polym. Phys. 45, 3078-3083 (2007)

$6 \mathrm{He}$, X. J., Du, J. H., Ying, Z. \& Cheng, M. Positive temperature coefficient effect in multiwalled carbon nanotube/high-density polyethylene composites. Appl. Phys. Lett. 86, 062112-062114 (2005).

7 Horibe, H., Kamimura, T. \& Yoshida, K. Electrical conductivity of polymer composites filled with metal. Jpn J. Appl. Phys. 44, 4171-4175 (2005).

8 Kono, A., Shimizu, K., Nakano, H., Goto, Y., Kobayashi, Y., Ougizawa, T. \& Horibe, H. Positive-temperaturecoefficient effect of electrical resistivity below melting point of poly(vinylidene fluoride) (PVDF) in $\mathrm{Ni}$ particle-dispersed PVDF composites. Polymer 53, 1760-1764 (2012)

9 Kono, A., Miyakawa, N., Kawadai, S., Goto, Y. Maruoka, T., Yamamoto, M. \& Horibe, H. Effect of cooling rate after polymer melting on electrical properties of high-density polyethylene/Ni composites. Polym J. 42, 587-591 (2010).

10 Kono, A., Kawadai, S., Yamamoto, M. \& Horibe, H. Relationship between crystallina of linear low-density polyethylene (LLDPE) and resistivity-temperature property of the LLDPE/Ni composites. Kobunshi Ronbunshu 67, 296-303 (2010)

11 Xu, H. P., Dang, Z. M., Bing, N. C., Wu, Y. H. \& Yang D. D. Temperature dependence of electric and dielectric behaviors of $\mathrm{Ni} /$ polyvinylidene fluoride composites. J. Appl. Phys. 107, 034105-04109 (2010).

12 Pike, G. E. \& Seager, C. H. Percolation and conductivity: a computer study. I. Phys. Rev. B 10, 1421-1434 (1973).

13 Balberg, I. Tunneling and nonuniversal conductivity in composite materials. Phys. Rev. Lett. 59, 1305-1308 (1987).

14 Straley, J. P. Critical exponents for the conductivity of random resistor lattices. Phys. Rev. B 15, 5733-5737 (1977).

15 Jeon, K., Lumata, L., Tokumoto, T., Steven, E., Brooks, J. \& Alamo, R. G. Low electrical conductivity threshold and crystalline morphology of single-walled carbon nanotubes-high density polyethylene nanocomposites characterized by SEM, Raman spectroscopy and AFM. Polymer 48, 4751-4764 (2007).

16 Nakamura, S., Saito, K., Sawa, G. \& Snarskii, A Critical exponent of conductivity and percolation phenomena of carbon black-polyethylene composites. Trans. IEEE Jpn 117-A, 371-380 (1997).

17 Sheng, P. Sichel, E. K \& Gittleman, J. I. Fluctuationinduced tunneling conduction in carbon-polyvinylchloride composites. Phys. Rev. Lett. 40, 1197-1200 (1978).

18 Wu, G., Asai, S., Zhang, C., Miura, T. \& Sumita, M. A delay of percolation time in carbon-black-filled conductive polymer composites. J. Appl. Phys. 88, 1480-1487 (2000) 